\title{
Study of the slepton non-universality at the CERN Large Hadron Collider
}

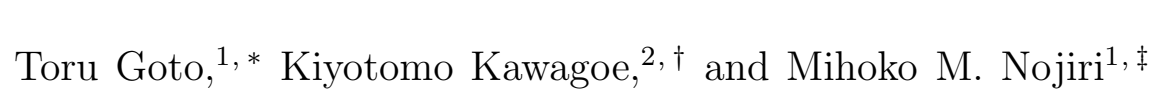 \\ ${ }^{1}$ YITP, Kyoto University, Kyoto 606-8502, Japan \\ ${ }^{2}$ Department of Physics, Kobe University, Kobe 657-8501, Japan
}

\begin{abstract}
In supersymmetric theory, the sfermion-fermion-gaugino interactions conserve the chirality of (s)fermions. The effect appears as the charge asymmetry in $m(j l)$ distributions at the CERN Large Hadron Collider where jets and leptons arise from the cascade decay $\tilde{q} \rightarrow q \tilde{\chi}_{2}^{0} \rightarrow q l \tilde{l}$. Furthermore, the decay branching ratios and the charge asymmetries in $m(j l)$ distributions are flavor nonuniversal due to the $\tilde{l}_{L}$ and $\tilde{l}_{R}$ mixing. When $\tan \beta$ is large, the non-universality between $e$ and $\mu$ becomes $O(10) \%$ level. We perform a Monte Carlo simulation for some minimal supergravity benchmark points to demonstrate the detectability.
\end{abstract}

PACS numbers: 11.30.Pb, 12.60.Jv, 14.80.Ly

*Electronic address: goto@yukawa.kyoto-u.ac.jp

${ }^{\dagger}$ Electronic address: kawagoe@phys.sci.kobe-u.ac.jp

${ }^{\ddagger}$ Electronic address: nojiri@yukawa.kyoto-u.ac.jp 


\section{INTRODUCTION}

Supersymmetry (SUSY) is one of the promising candidates of the physics beyond the standard model. No signature of SUSY has been found yet. However, the discovery of the SUSY particles is guaranteed for $m_{\tilde{q}}, m_{\tilde{g}} \lesssim 2.5 \mathrm{TeV}$ at the CERN Large Hadron Collider (LHC) for the minimal supergravity (mSUGRA) model. Date taking is expected to start from 2007. Masses of the sparticles will also be measured at the LHC [1] with reasonable accuracy, which is important to distinguish various SUSY breaking models.

The Lagrangian of supersymmetric theory is highly constrained. For example the sfermion-fermion-gaugino interaction is restricted to be of the form $\tilde{f}_{L(R)^{-}} f_{L(R)}-\tilde{G}$ because $\tilde{f}$ and $f$ belong to the same chiral multiplet in supersymmetry. On the other hand, $\tilde{f}_{L}$ and $\tilde{f}_{R}$ mixing terms are controlled by the $F$ term of the superpotential of the form $W=\mu H_{1} H_{2}+y_{u} Q U^{c} H_{2}+y_{d} Q D^{c} H_{1}+y_{l} L E^{c} H_{1}$. Therefore the mixing term is proportional to the Yukawa coupling $y_{f}$ and $\mu$ parameter. Studying such mixing terms determined by the supersymmetry would be an interesting target for collider physics after the discovery of the supersymmetry.

In this paper we study the effect of the neutralino polarization in the decay chain

$$
\tilde{q} \rightarrow q \tilde{\chi}_{2}^{0} \rightarrow q l_{1}^{ \pm} \tilde{l}^{\mp} \rightarrow q l_{1}^{ \pm} l_{2}^{\mp} \tilde{\chi}_{1}^{0}
$$

here $l_{1}\left(l_{2}\right)$ denotes the lepton from $\tilde{\chi}_{2}^{0}(\tilde{l})$ decay. Due to the chirality structure of the squarkquark-neutralino coupling, the $\tilde{\chi}_{2}^{0}$ is polarized. The polarization of $\tilde{\chi}_{2}^{0}$ then affects the

angular distribution of the slepton in the $\tilde{\chi}_{2}^{0} \rightarrow l_{1}^{ \pm} \tilde{l}^{\mp}$ decay. The polarization dependence of the angular distribution eventually shows up in the charge asymmetry in the $m\left(q l_{1}^{ \pm}\right)$ distribution, because the polarization dependent part of the amplitude flips under the charge conjugation transformation. The effect can be seen at the $p p$ collider LHC because the number of squarks $N(\tilde{q})$ produced at the LHC is larger than $N\left(\tilde{q}^{*}\right)$. The polarization effect is considered in Refs. [2, 3], where the slepton is assumed to be purely right-handed. We study this process taking account of the left-right mixing of the sleptons, which depends on the lepton flavor. We point out that the left-right mixing significantly affects the charge asymmetry as well as the decay width of $\tilde{\chi}_{2}^{0} \rightarrow l_{1}^{ \pm} \tilde{l}^{\mp}$ for $l=\mu$ and $\tau$.

This paper is organized as follows. In the next section, we first summarize the relevant formulae and discuss the effect of the left-right mixing qualitatively, then we evaluate the 
lepton flavor dependence of the charge asymmetry and the decay widths for explicit examples. In Sec. III we carry out the Monte Carlo (MC) study and discuss the feasibility to study the chiral effect at the LHC. Sec. IV is devoted for discussions.

\section{SQUARK CASCADE DECAY AND LEPTON CHARGE ASYMMETRY}

\section{A. Formalism}

We parameterize the sfermion-fermion-neutralino interaction Lagrangian as

$$
\mathcal{L}=-\frac{g_{2}}{\sqrt{2}} \sum_{i, \alpha} \overline{\tilde{\chi}}_{i}^{0}\left(L_{i \alpha}^{f} \frac{1-\gamma_{5}}{2}+R_{i \alpha}^{f} \frac{1+\gamma_{5}}{2}\right) f \tilde{f}_{\alpha}^{*}+\text { H.c. },
$$

where $i=1,2,3,4$ and $\alpha=1,2$ are the suffices for the mass eigenstates of the neutralinos and the sfermions, respectively. $g_{2}$ is the $\mathrm{SU}(2)$ gauge coupling constant. For the charged lepton sector, the coupling constants $L_{i \alpha}^{l}$ and $R_{i \alpha}^{l}$ are given as

$$
\begin{aligned}
L_{i 1}^{l} & =-\left[\left(U_{N}^{*}\right)_{i 2}+\left(U_{N}^{*}\right)_{i 1} \tan \theta_{W}\right] \cos \theta_{l}+\frac{m_{l}}{m_{W} \cos \beta}\left(U_{N}^{*}\right)_{i 3} \sin \theta_{l}, \\
L_{i 2}^{l} & =\left[\left(U_{N}^{*}\right)_{i 2}+\left(U_{N}^{*}\right)_{i 1} \tan \theta_{W}\right] \sin \theta_{l}+\frac{m_{l}}{m_{W} \cos \beta}\left(U_{N}^{*}\right)_{i 3} \cos \theta_{l}, \\
R_{i 1}^{l} & =2\left(U_{N}\right)_{i 1} \tan \theta_{W} \sin \theta_{l},+\frac{m_{l}}{m_{W} \cos \beta}\left(U_{N}\right)_{i 3} \cos \theta_{l} \\
R_{i 2}^{l} & =2\left(U_{N}\right)_{i 1} \tan \theta_{W} \cos \theta_{l},-\frac{m_{l}}{m_{W} \cos \beta}\left(U_{N}\right)_{i 3} \sin \theta_{l}
\end{aligned}
$$

where $U_{N}$ is the unitary matrix which diagonalize the neutralino mass matrix. The left-right mixing angle of the sleptons $\theta_{l}$ is defined as follows. The slepton mass term is written as

$$
-\mathcal{L}_{\text {mass }}(\tilde{l})=\left(\begin{array}{cc}
\tilde{l}_{L}^{*} & \tilde{l}_{R}^{*}
\end{array}\right) \mathcal{M}_{\tilde{l}}^{2}\left(\begin{array}{c}
\tilde{l}_{L} \\
\tilde{l}_{R}
\end{array}\right)
$$

where the mass squared matrix $\mathcal{M}_{\tilde{l}}^{2}$ is given as

$$
\mathcal{M}_{\tilde{l}}^{2}=\left(\begin{array}{cc}
m_{\tilde{l}_{L}}^{2}+m_{l}^{2}+m_{Z}^{2} \cos 2 \beta\left(\sin ^{2} \theta_{W}-\frac{1}{2}\right) & -m_{l}\left(A_{l}^{*}+\mu \tan \beta\right) \\
-m_{l}\left(A_{l}+\mu^{*} \tan \beta\right) & m_{\tilde{l}_{R}}^{2}+m_{l}^{2}-m_{Z}^{2} \cos 2 \beta \sin ^{2} \theta_{W}
\end{array}\right) .
$$

In this paper, we neglect lepton flavor mixing and assume that $A_{l}$ and $\mu$ are real. Then $\theta_{l}$ is obtained by diagonalizing the slepton mass matrix:

$$
U \mathcal{M}_{\tilde{l}}^{2} U^{\dagger}=\left(\begin{array}{cc}
m_{\tilde{l}_{1}}^{2} & 0 \\
0 & m_{\tilde{l}_{2}}^{2}
\end{array}\right), \quad U=\left(\begin{array}{cc}
\cos \theta_{l} & \sin \theta_{l} \\
-\sin \theta_{l} & \cos \theta_{l}
\end{array}\right)
$$


where we assume $m_{\tilde{l}_{1}}^{2}<m_{\tilde{l}_{2}}^{2}$. The mass eigenstates $\tilde{l}_{1,2}$ are defined as

$$
\left(\begin{array}{l}
\tilde{l}_{1} \\
\tilde{l}_{2}
\end{array}\right)=U\left(\begin{array}{l}
\tilde{l}_{L} \\
\tilde{l}_{R}
\end{array}\right)
$$

The coupling constants for the squark sector are obtained in the same way.

The decay widths of the subprocesses $\tilde{f}_{\alpha} \rightarrow f \tilde{\chi}_{i}^{0}$ and $\tilde{\chi}_{i}^{0} \rightarrow l^{ \pm} \tilde{l}_{\alpha}^{\mp}$ are written as

$$
\begin{aligned}
\Gamma\left(\tilde{f}_{\alpha} \rightarrow f \tilde{\chi}_{i}^{0}\right) & =\frac{g_{2}^{2} m_{\tilde{f}_{\alpha}}}{32 \pi}\left(1-\frac{m_{\tilde{\chi}_{i}^{0}}^{2}}{m_{\tilde{f}_{\alpha}}^{2}}\right)^{2}\left(\left|L_{i \alpha}^{f}\right|^{2}+\left|R_{i \alpha}^{f}\right|^{2}\right), \\
\Gamma\left(\tilde{\chi}_{i}^{0} \rightarrow l^{ \pm} \tilde{l}_{\alpha}^{\mp}\right) & =\frac{g_{2}^{2} m_{\tilde{\chi}_{i}^{0}}}{64 \pi}\left(1-\frac{m_{\tilde{l}_{\alpha}}^{2}}{m_{\tilde{\chi}_{i}^{0}}^{2}}\right)^{2}\left(\left|L_{i \alpha}^{l}\right|^{2}+\left|R_{i \alpha}^{l}\right|^{2}\right) .
\end{aligned}
$$

Here and hereafter, we neglect the quark and lepton masses for the kinematics, while keeping them in the coupling constants Eqs. (3)-(6)

The momentum configuration of the whole decay chain $\tilde{q}_{\beta} \rightarrow q \tilde{\chi}_{2}^{0} \rightarrow q l_{1}^{ \pm} \tilde{l}_{\alpha}^{\mp} \rightarrow q l_{1}^{ \pm} l_{2}^{\mp} \tilde{\chi}_{1}^{0}$ is described by three angular variables. The distribution of the decay chain is given as

$$
\begin{aligned}
\frac{d^{3} \Gamma}{d \cos \theta_{\tilde{l}} d \cos \theta_{\tilde{\chi}_{1}^{0}} d \phi_{\tilde{\chi}_{1}^{0}}}= & \frac{1}{8 \pi} \Gamma\left(\tilde{q}_{\beta} \rightarrow q \tilde{\chi}_{2}^{0}\right) \operatorname{Br}\left(\tilde{\chi}_{2}^{0} \rightarrow l_{1}^{ \pm} \tilde{l}_{\alpha}^{\mp}\right) \operatorname{Br}\left(\tilde{l}_{\alpha}^{\mp} \rightarrow l_{2}^{\mp} \tilde{\chi}_{1}^{0}\right) \\
& \times\left[1 \mp A(l) \cos \theta_{\tilde{l}}\right] \\
A(l)= & \frac{\left|L_{2 \beta}^{q}\right|^{2}-\left|R_{2 \beta}^{q}\right|^{2}}{\left|L_{2 \beta}^{q}\right|^{2}+\left|R_{2 \beta}^{q}\right|^{2}} \cdot \frac{\left|L_{2 \alpha}^{l}\right|^{2}-\left|R_{2 \alpha}^{l}\right|^{2}}{\left|L_{2 \alpha}^{l}\right|^{2}+\left|R_{2 \alpha}^{l}\right|^{2}},
\end{aligned}
$$

where $\operatorname{Br}$ denotes the branching ratio $\operatorname{Br}\left(\tilde{\chi}_{2}^{0} \rightarrow l^{ \pm} \tilde{l}_{\alpha}^{\mp}\right)=\Gamma\left(\tilde{\chi}_{2}^{0} \rightarrow l^{ \pm} \tilde{l}_{\alpha}^{\mp}\right) / \Gamma_{\text {total }}$, etc. $\theta_{\tilde{l}}$ is the angle between the momenta of the quark and the lepton $l_{1}$ in the $\tilde{\chi}_{2}^{0}$ rest frame, $\theta_{\tilde{\chi}_{1}^{0}}$ is the angle between the two lepton momenta in the slepton rest frame, and $\phi_{\tilde{\chi}_{1}^{0}}$ is the angle between the decay planes of $\tilde{q} \rightarrow q l_{1}^{ \pm} \tilde{l}^{\mp}$ and $\tilde{\chi}_{2}^{0} \rightarrow l_{1}^{ \pm} l_{2}^{\mp} \tilde{\chi}_{1}^{0}$. Since squark and slepton decays are spherically symmetric in the rest frames of the decaying particles, the angular distribution is flat over $\cos \theta_{\tilde{\chi}_{1}^{0}}$ and $\phi_{\tilde{\chi}_{1}^{0}}$. The $\theta_{\tilde{l}}$ dependence comes from the polarization of $\tilde{\chi}_{2}^{0}$ and the chirality structure of the sfermion-fermion-neutralino interaction shows up in this angular distribution. Qualitatively if $\tilde{q}_{L}$ decays into $\tilde{\chi}_{2}^{0}$ followed by $\tilde{\chi}_{2}^{0} \rightarrow l^{+} \tilde{l}^{-}$, the lepton favors going in the same (opposite) direction to $\tilde{\chi}_{2}^{0}$ for $\tilde{l}_{L(R)}$.

Instead of the above angular variables, we consider the invariant masses of the combinations of the quark and the leptons, which are directly connected as the observables. The 
relations are given as

$$
\begin{aligned}
m^{2}\left(l_{1} l_{2}\right)= & \frac{\left(m_{\tilde{\chi}_{2}^{0}}^{2}-m_{\tilde{l}}^{2}\right)\left(m_{\tilde{l}}^{2}-m_{\tilde{\chi}_{1}^{0}}^{2}\right)}{m_{\tilde{l}}^{2}} \frac{1-\cos \theta_{\tilde{\chi}_{1}^{0}}}{2} \\
m^{2}\left(q l_{1}\right)= & \frac{\left(m_{\tilde{q}}^{2}-m_{\tilde{\chi}_{2}^{0}}^{2}\right)\left(m_{\tilde{\chi}_{2}^{0}}^{2}-m_{\tilde{l}}^{2}\right)}{m_{\tilde{\chi}_{2}^{0}}^{2}} \frac{1-\cos \theta_{\tilde{l}}}{2}, \\
m^{2}\left(q l_{2}\right)= & \frac{1}{4} \frac{\left(m_{\tilde{q}}^{2}-m_{\tilde{\chi}_{2}^{0}}^{2}\right)\left(m_{\tilde{l}}^{2}-m_{\tilde{\chi}_{1}^{0}}^{2}\right)}{m_{\tilde{\chi}_{2}^{0}}^{2} m_{\tilde{l}}^{2}} \\
& \times\left[m_{\tilde{\chi}_{2}^{0}}^{2}\left(1+\cos \theta_{\tilde{l}}\right)\left(1-\cos \theta_{\tilde{\chi}_{1}^{0}}\right)+m_{\tilde{l}}^{2}\left(1-\cos \theta_{\tilde{l}}\right)\left(1+\cos \theta_{\tilde{\chi}_{1}^{0}}\right)\right. \\
& \left.+2 m_{\tilde{\chi}_{2}^{0}} m_{\tilde{l}} \sin \theta_{\tilde{l}} \sin \theta_{\tilde{\chi}_{1}^{0}} \cos \phi_{\tilde{\chi}_{1}^{0}}\right]
\end{aligned}
$$

The polarization effect of $\tilde{\chi}_{2}^{0}$ can be seen clearly in the charge asymmetry in the $m\left(q l_{1}^{ \pm}\right)$ distribution, since an $A(l)$ is the coefficient of $\theta_{\tilde{l}}$, and it affects the decay distributions for $\tilde{q} \rightarrow q \tilde{\chi}_{2}^{0} \rightarrow q l_{1}^{+} \tilde{l}^{-}$and $\tilde{q} \rightarrow q \tilde{\chi}_{2}^{0} \rightarrow q l_{1}^{-} \tilde{l}^{+}$with opposite signs. However, the leptons $l_{1}$ of the neutralino decay are indistinguishable from $l_{2}$ of the slepton decay. We therefore study the charge asymmetry between the $m\left(q l^{ \pm}\right)$distributions taking both $l_{1}$ and $l_{2}$ into account. The $m\left(q l^{+}\right)$distribution is the sum of the $m\left(q l_{1}^{+}\right)$distribution from the decay chain $\tilde{\chi}_{2}^{0} \rightarrow l_{1}^{+} \tilde{l}^{-} \rightarrow l_{1}^{+} l_{2}^{-} \tilde{\chi}_{1}^{0}$ and the $m\left(q l_{2}^{+}\right)$distribution from $\tilde{\chi}_{2}^{0} \rightarrow l_{1}^{-} \tilde{l}^{+} \rightarrow l_{1}^{-} l_{2}^{+} \tilde{\chi}_{1}^{0}$.

The charge asymmetry in $m\left(q l^{ \pm}\right)$distribution was studied in Ref. [3] in a case where the slepton is purely right-handed: $L_{2 \alpha}^{l}=0$. In this paper we take account of the left-right mixing of the sleptons, whose effect depends on the lepton flavor.

\section{B. Effect of the slepton left-right mixing in mSUGRA model}

We consider a "typical" case of the minimal supergravity model. The SU(2) and U(1) gaugino masses $M_{2}$ and $M_{1}$, respectively, are related to each other as $M_{1} \approx 0.5 M_{2}$ and the magnitude of the Higgsino mass $\mu$ is assumed to be larger than $M_{2}$, so that the wino component dominates $\tilde{\chi}_{2}^{0}$ and the bino component dominates $\tilde{\chi}_{1}^{0}$. As for the slepton mass matrix, the right-handed slepton mass parameter becomes smaller than the left-handed one due to the running effect. Therefore the lighter slepton, $\tilde{l}_{1}$, is $\tilde{l}_{R}$-like and the heavier one, $\tilde{l}_{2}$, is $\tilde{l}_{L}$-like in the most of the parameter space.

We can safely neglect the left-right mixing of the squarks because the process we consider is the decay of the first generation squark. The first decay process $\tilde{q} \rightarrow q \tilde{\chi}_{2}^{0}$ in Eq. (11) occurs 
predominantly through the $\tilde{q}_{L^{-}} q-\tilde{W}$ coupling if $\tilde{\chi}_{2}^{0} \sim \tilde{W}$. Consequently the first factor of the right-hand side of Eq. (14) is very close to unity.

Let us now consider the decay process $\tilde{\chi}_{2}^{0} \rightarrow l^{ \pm} \tilde{l}_{1}^{\mp}$ with $l=e, \mu$, or $\tau$. We first note that, in the minimal supergravity model, the slepton left-right mixing angle $\theta_{l}$ is approximately written as

$$
\tan 2 \theta_{l} \approx-\frac{m_{l}\left(A_{l}+\mu \tan \beta\right)}{0.37 M_{1 / 2}^{2}}\left(1+O\left(\frac{m_{0}^{2} m_{l}^{2} \tan ^{2} \beta}{M_{1 / 2}^{2} m_{W}^{2}}\right)\right),
$$

where $m_{0}$ and $M_{1 / 2}$ are the universal scalar mass and the gaugino mass at the GUT scale, respectively, and $A_{l}$ is the trilinear scalar coupling constant at the low energy scale. The numerical factor 0.37 in the denominator is determined by the gauge coupling constants. In the (s)electron case, the left-right mixing of the selectrons is negligible, $i . e ., \cos \theta_{l} \approx 0$ for $l=e$ in Eqs. (3) -(6) , and the charge asymmetry is maximal $(A(e) \approx-1)$.

The effect of the left-right mixing and the Yukawa coupling is quite significant for the (s)tau mode. For $l=\tau$, the typical magnitude of $\cos \theta_{\tau}$ is $O(1)$ for large $\tan \beta$ in mSUGRA. Magnitudes of the neutralino mixing matrix elements are typically $\left|\left(U_{N}\right)_{22}\right| \approx 1$ and $\left|\left(U_{N}\right)_{21,23}\right|=O\left(10^{-1}\right)$. Substituting these numbers in Eqs. (3) and (15), we see that $L_{21}^{\tau}$ can dominate over $R_{21}^{\tau}$ and the behavior of the charge asymmetry is opposite to the electron case.

The left-right mixing effect may be observed even in the (s)muon case. Since both terms of the right-hand side of Eq. (3) are approximately proportional to the lepton mass, $L_{21}^{\mu}$ is enhanced by $O\left(m_{\mu} / m_{e}\right)$ compared to $L_{21}^{e}$. For relatively large $\tan \beta$, it is possible that $L_{21}^{\mu}$ and $R_{21}^{\mu}$ are of the same order of magnitude, as we will see later.

Notice that the branching ratio of $\tilde{\chi}_{2}^{0} \rightarrow l \tilde{l}_{1}$ also depends on the lepton flavor. The coupling $R_{21}^{l}$ is suppressed due to the small $\left(U_{N}\right)_{21}$ component, therefore even tiny mixing of the left-hand component will have a significant effect. Especially, the decay width for $\tau \tilde{\tau}_{1}$ mode is much larger than those for $e \tilde{e}_{1}$ and $\mu \tilde{\mu}_{1}$, and $\Gamma\left(\tilde{\chi}_{2}^{0} \rightarrow \mu \tilde{\mu}_{1}\right)>\Gamma\left(\tilde{\chi}_{2}^{0} \rightarrow e \tilde{e}_{1}\right)$.

We also consider the case where $m_{\tilde{\chi}_{2}^{0}}>m_{\tilde{l}_{2}}$. In this case, both $\tilde{l}_{1}$ and $\tilde{l}_{2}$ contribute to the decay chain so that the decay distribution over $m\left(q l^{ \pm}\right)$is more complicated. Since $\tilde{l}_{2}$ is $\tilde{l}_{L}$-like, the coupling $L_{22}^{l}$ in Eq. (41) dominates the $\tilde{\chi}_{2}^{0} \rightarrow l^{ \pm} \tilde{l}_{2}^{\mp}$ decay and the branching ratio of $\tilde{\chi}_{2}^{0} \rightarrow l^{ \pm} \tilde{l}_{2}^{\mp}$ is much larger than that of $\tilde{\chi}_{2}^{0} \rightarrow l^{ \pm} \tilde{l}_{1}^{\mp}$. The main contribution of the charge asymmetry therefore comes from $\tilde{l}_{2}$.

Let us study the lepton flavor dependence more quantitatively. In order to compare with 


\begin{tabular}{|c||c|c|c|c|c|c|c|c|}
\hline model & $m_{\tilde{u}_{L}}$ & $m_{\tilde{\chi}_{2}^{0}}$ & $m_{\tilde{\chi}_{1}^{0}}$ & $m_{\tilde{l}}$ & $m_{q l_{1}}$ & $m_{q l_{2}}$ & $m_{q l l}$ & $m_{l l}$ \\
\hline SPS1a & 537.3 & 176.8 & 96.1 & 143.0 & 298.4 & 375.7 & 425.9 & 77.0 \\
SPS3 $\left(\tilde{l}_{R}\right)$ & 832.4 & 303.7 & 164.0 & 182.3 & 619.9 & 310.2 & 652.3 & 106.1 \\
$\operatorname{SPS} 3\left(\tilde{l}_{L}\right)$ & & & & 292.8 & 208.8 & 642.0 & 652.3 & 66.8 \\
\hline
\end{tabular}

TABLE I: The sparticle masses and end points of $q l l$ distributions arising from the cascade decay $\tilde{q} \rightarrow q \tilde{\chi}_{2}^{0} \rightarrow q l_{1} \tilde{l} \rightarrow q l_{1} l_{2} \tilde{\chi}_{1}^{0}$ at SPS1a and SPS3. The masses and end points for SPS1a $(\tan \beta=15$, 20) are similar to that for $\tan \beta=10$ for the first and second generation sfermions. All masses are given in $\mathrm{GeV}$.

the Monte Carlo simulations in the next section, we take benchmark points SPS1a and SPS3 given in Ref. [4], where the mass spectrum is calculated by ISAJET [6]. The model points we study are as follows;

- SPS1 [4]:

This is the mSUGRA point where $m_{0}=100 \mathrm{GeV}, M_{1 / 2}=250 \mathrm{GeV}$, the trilinear coupling at the GUT scale $A_{0}=-100 \mathrm{GeV}, \tan \beta=10$ and $\mu>0$. The decay $\tilde{\chi}_{2}^{0} \rightarrow \tilde{l}_{R} l$ is open. We also show some results for the point where $m_{0}$ and $M_{1 / 2}$ are the same as those of SPS1a but $\tan \beta=15$ and 20. For these points, the effect of $\tilde{\mu}$ left-right mixing in $\tilde{\chi}_{2}^{0}$ decay becomes significant.

- SPS3 (or point C of [5]):

$m_{0}=90 \mathrm{GeV}, M_{1 / 2}=400 \mathrm{GeV}, A_{0}=0, \tan \beta=10$ and $\mu>0$. The decay $\tilde{\chi}_{2}^{0} \rightarrow \tilde{l}_{L} l$ is open for this point. The decay must show opposite charge asymmetry to that of $\tilde{l}_{R}$.

The kinematics of the decay distribution is important for our study. We therefore list the calculated end points for the decay (11) in Table I.

Since the masses and the Yukawa couplings of the first and second generations are neglected in ISAJET, its output cannot be used directly for the discussion of $e-\mu$ difference. We take account of the left-right mixings of selectrons and smuons in the following way. The output from ISAJET contains a set of the low energy MSSM parameters $m_{\tilde{q}_{L}}, m_{\tilde{d}_{R}}$, $m_{\tilde{u}_{R}}, m_{\tilde{l}_{L}}$ and $m_{\tilde{e}_{R}}$ for the first generation sfermions, and $m_{\tilde{q}_{3 L}}, m_{\tilde{b}_{R}}, m_{\tilde{t}_{R}}, m_{\tilde{l}_{3 L}}, m_{\tilde{\tau}_{R}}, A_{t}$, $A_{b}$ and $A_{\tau}$ for the third generation sfermions, as well as the parameters in the gaugino and Higgs(ino) sectors. The soft SUSY breaking parameters for the second generation sfermions 
are assumed to be the same as the first generation ones. Since the trilinear couplings $A_{e, \mu}$ are not given in the ISAJET output, we take $A_{e}=A_{\mu}=A_{\tau}$, but the left-right mixing mainly comes from the $\mu \tan \beta$ term anyway. Then we diagonalize the mass matrix (8) to obtain the mixing angle $\theta_{l}$ in (9) and evaluate the coupling constants $L_{i \alpha}^{l}$ and $R_{i \alpha}^{l}$. The slepton mass eigenvalues obtained with this procedure are close to the original ISAJET output. The numerical values of the coupling constants relevant to the $\tilde{\chi}_{2}^{0} \rightarrow l \tilde{l}_{\alpha}$ are given in Table $\amalg$. ¿From these values, we obtain

$$
\begin{aligned}
& \Gamma(e): \Gamma(\mu): \Gamma(\tau) \approx 1: 1.04: 13.7 \\
& A(e): A(\mu): A(\tau) \approx 1: 0.93:-0.84
\end{aligned}
$$

\begin{tabular}{|c|c|c|c|c|c|}
\hline Model & $l$ & $\left|L_{21}^{l}\right|$ & $\left|R_{21}^{l}\right|$ & $\left|L_{22}^{l}\right|$ & $\left|R_{22}^{l}\right|$ \\
\hline \multirow[t]{3}{*}{ SPS1a } & $e$ & $.06 \times 10^{-4}$ & 0.116 & 0.999 & $2.81 \times 10^{-5}$ \\
\hline & $\mu$ & 0.0219 & 0.116 & 0.998 & $5.81 \times 10^{-3}$ \\
\hline & $\tau$ & 0.327 & 0.0950 & 0.946 & 0.0909 \\
\hline \multirow[t]{3}{*}{ SPS3 } & & $.47 \times 10^{-5}$ & 0.0598 & 0.990 & $1.77 \times 10^{-5}$ \\
\hline & $\mu$ & 0.0134 & 0.0598 & 0.990 & $3.66 \times 10^{-3}$ \\
\hline & $\tau$ & 0.215 & 0.0505 & 0.968 & 0.0603 \\
\hline
\end{tabular}

for the SPS1a. Here $\Gamma(l)$ denotes the decay width $\Gamma\left(\tilde{\chi}_{2}^{0} \rightarrow l^{ \pm} \tilde{l}_{1}^{\mp}\right)$.

TABLE II: Magnitude of slepton-lepton-neutralino coupling constants for the benchmark points SPS1a and SPS3.

We see that the effect of the smuon left-right mixing is rather small in the above model points, where $\tan \beta=10$. However, $L_{21}^{l}$ is enhanced for larger $\tan \beta$ since the source of the slepton left-right mixing is the Yukawa coupling, while $R_{21}^{l}$, whose main component is the $\mathrm{U}(1)$ gauge coupling, is less sensitive to $\tan \beta$. Therefore, we expect a larger $e-\mu$ difference in $\Gamma(l)$ and $A(l)$ for larger $\tan \beta$. In order to see the $\tan \beta$ dependence, we consider variants of SPS1a, where the mSUGRA parameters have the same values as those in SPS1a, but $\tan \beta$ is taken to be different. The relative magnitudes of the decay widths and $A(l)$, as well as the magnitudes of relevant coupling constants for $\tan \beta=10,15$ and 20 are listed in Table III. We see $\left|L_{21}^{\mu}\right|$ is proportional to $\tan \beta$ in this region and the $e-\mu$ differences in $\Gamma(l)$ and $A(l)$ are quite significant for $\tan \beta=20$. 


\begin{tabular}{cccccc}
\hline $\tan \beta$ & $l$ & $\left|L_{21}^{l}\right|$ & $\left|R_{21}^{l}\right|$ & $\Gamma(l) / \Gamma(e)$ & $A(l) / A(e)$ \\
\hline 10 & $e$ & $1.06 \times 10^{-4}$ & 0.116 & 1 & 1 \\
& $\mu$ & 0.0219 & 0.116 & 1.04 & 0.93 \\
& $\tau$ & 0.327 & 0.0950 & 13.7 & -0.84 \\
\hline 15 & $e$ & $1.58 \times 10^{-4}$ & 0.107 & 1 & 1 \\
& $\mu$ & 0.0326 & 0.107 & 1.09 & 0.83 \\
& $\tau$ & 0.438 & 0.0671 & 37.6 & -0.95 \\
\hline 20 & $e$ & $2.09 \times 10^{-4}$ & 0.102 & 1 & 1 \\
& $\mu$ & 0.0432 & 0.102 & 1.17 & 0.70 \\
& $\tau$ & 0.522 & 0.0419 & 80.2 & -0.99 \\
\hline
\end{tabular}

TABLE III: Absolute values of the coupling constants and relative magnitudes of the decay widths and $A(l)$ for different choices of $\tan \beta$. The other mSUGRA parameters are same as those in SPS1a.

Besides the slepton-lepton-neutralino coupling constants, the mass spectrum is also affected by changing the value of $\tan \beta$. However, since the shifts are tiny for $m_{\tilde{e}_{1}}, m_{\tilde{\mu}_{1}}$ and $m_{\tilde{\chi}_{2}^{0}}$, the $\tan \beta$ dependence of the $e-\mu$ difference in the decay width and the charge asymmetry comes dominantly from the $\tan \beta$ dependence of the $\tilde{\mu}$ left-right mixing angle. On the other hand, the mass eigenvalue of $\tilde{\tau}_{1}$ changes substantially: $133 \mathrm{GeV}$ for $\tan \beta=10$ to 108 $\mathrm{GeV}$ for $\tan \beta=20$, namely. Consequently, the $e^{-\tau} \tau$ differences and the $\tan \beta$ dependence in Table III are the cumulative effect of both the mixing angle and the mass eigenvalue.

\section{SIMULATIONS}

\section{A. Charge asymmetry}

In this section, we show some simulation results at SPS1a and SPS3 and discuss the LHC potential to study the chiral nature of sfermions at the LHC in the decay (10).

We generated $3 \times 10^{6}$ events for SPS1a $(\tan \beta=10)$ and SPS3. This corresponds to $\int \mathcal{L} d t=58 \mathrm{fb}^{-1}$ for SPS1a and $600 \mathrm{fb}^{-1}$ for SPS3 respectively. The mass spectrum and branching ratios are generated by ISAJET [6] and interfaced to HERWIG [7], which means the effect of the non-universal branching ratios of $\tilde{\chi}_{2}^{0} \rightarrow \tilde{e} e$ and $\tilde{\mu} \mu$ discussed in the previous section is not taken into account in our event generations. The events are studied using the 


$$
\begin{aligned}
& n_{j}\left(P_{T}>100 \mathrm{GeV}\right) \geq 1 \\
& n_{j}\left(P_{T}>50 \mathrm{GeV}\right) \geq 4 \\
& n_{\mathrm{b} \text { jet }}\left(P_{T}>50\right)=0 \\
& m_{\mathrm{eff}}>600 \mathrm{GeV}, \text { where } m_{\mathrm{eff}}=p_{\text {miss }}^{T}+p_{j 1}^{T}+p_{j 2}^{T}+p_{j 3}^{T}+p_{j 4}^{T} \\
& E_{T}(\text { miss })>0.2 m_{\mathrm{eff}} \\
& \text { Two and only two opposite sign isolated leptons } \\
& P_{T l_{1}}>20 \mathrm{GeV}, P_{T l_{2}}>10 \mathrm{GeV}
\end{aligned}
$$

TABLE IV: Selection cuts used for the simulations in this paper.

fast detector simulator ATLFAST [8]. Cuts for the SUSY event selection are listed in Table IV]

The dominant production processes are $p p \rightarrow \tilde{g} \tilde{g}$ and $\tilde{g} \tilde{q}$ at the LHC. The production cross section for the first generation squarks are larger than that of anti-squarks, because the LHC is a $p p$ collider. On the other hand, a gluino decay is charge symmetric, therefore the squarks and anti-squarks from gluino decays dilute the total charge asymmetry of SUSY signal. For this simulation, we especially veto the events containing $b$ jets with $P_{T}>50 \mathrm{GeV}$ as they dominantly come from the decays $\tilde{g} \rightarrow \tilde{b} b$ and $\tilde{t} t$. For the points studied in this paper, more than $40 \%$ of the gluino decay involves $b$ jets. After applying the cuts in Table IV the number of events which involves $\tilde{u}_{L}$ or $\tilde{d}_{L}$ direct production are $50(58) \%$ of the total events for SPS1a(SPS3) respectively.

We have not performed a detailed simulation of the Standard Model background for this study. The applied cuts are however very similar to the cuts applied in [11], where the SM background is shown to be negligible with respect to the SUSY background. For the SPS3 Point, for which the signal statistics is lower and we perform the study for an integrated luminosity of $600 \mathrm{pb}^{-1}$, the SM background could be approximately at the level of the SUSY background. The subtraction procedure used for the SUSY background does work for the SM background which is dominated by $\bar{t} t$ production. Therefore we expect no significant modification of the result for SPS1a, and only a moderate reduction of the significance for SPS3, due to increased statistical fluctuation.

In Fig. 1] and 2, we show some of the $j l^{+} l^{-}$distribution at $\operatorname{SPS} 1 \mathrm{a}(\tan \beta=10)$ and SPS3. In Fig. 1 we show $m(l l)$ distributions, where we subtract the distributions of the 


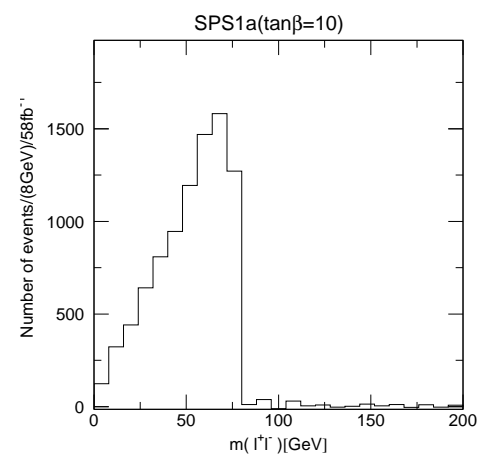

a)

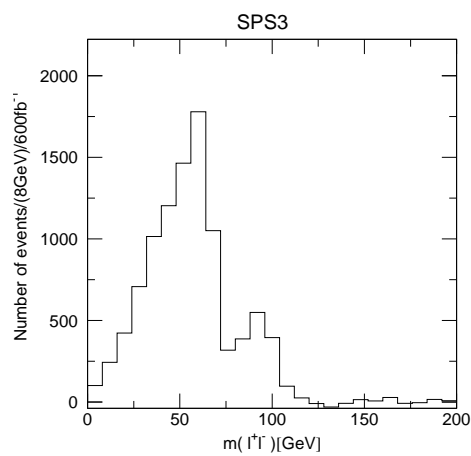

b)

FIG. 1: $m(l l)$ distributions for a) SPS1a and b) SPS3.

events with odd sign odd flavor leptons (OSOF, $e^{+} \mu^{-}$and $\mu^{+} e^{-}$) from the events with odd sign same flavor leptons (OSSF, $e^{+} e^{-}$and $\left.\mu^{+} \mu^{-}\right)$. The subtraction is known as a way to remove the backgrounds where two leptons are originating from $W$ or $\tau$ decays. Different detection efficiencies of electrons and muons may affect the subtraction, generating a systematic uncertainty in the result. The evaluation of these uncertainties is a complex experimental task, outside the scope of this study. We will therefore in the following only quote statistical errors. We can however note that the experiments will be able to exploit a very large sample of $W \rightarrow l \nu$ and $Z \rightarrow l^{+} l^{-}$decays to understand in detail the acceptance and efficiency for electrons and muons.

The $m(l l)$ distribution for the decay Eq. (11) has an edge structure at $m(l l, \max )$, which are reconstructed for both of the model points. Especially two edges are seen for SPS3, and the lighter one is consistent with the lepton pairs from the decay $\tilde{\chi}_{2}^{0} \rightarrow \tilde{l}_{L}, m(l l, \max )=$ $66.8 \mathrm{GeV}$.

We study the distribution of the two leptons and one of the two highest $p_{T}$ jets, as one of the two jets likely comes from $\tilde{q}$ decays if the mass difference between $\tilde{q}$ and $\tilde{\chi}_{2}^{0}$ is large enough. We denote the two jets as $j_{S}$ and $j_{L}$ where $m\left(j_{S} l l\right)<m\left(j_{L} l l\right)$. The clear endpoints are seen by taking $m\left(j_{S} l l\right)$ distributions as can be seen in Fig 2 ,

The $m\left(j_{S} l\right)$ distributions are shown in Fig. 3 a) and b). When the decay Eq. (11) is open, the $m\left(q l_{1}\right)$ distribution has an edge. The edge is very close to $m(j l, \max )$ for SPS1a, while it is much lower than $m(j l, \max )$ for SPS3, see Table【. This is because $\left(m_{\tilde{\chi}_{2}^{0}}-m_{\tilde{l}}\right) / m_{\tilde{\chi}_{2}^{0}} \ll 1$ 


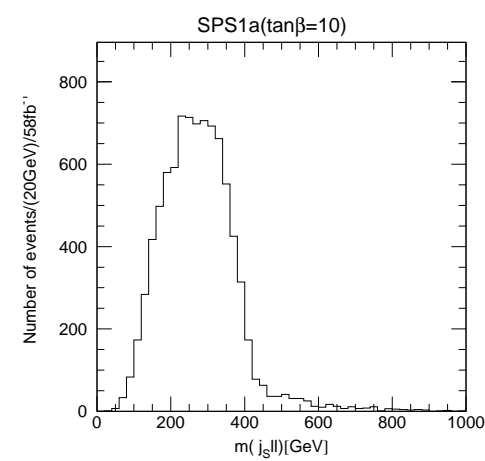

a)

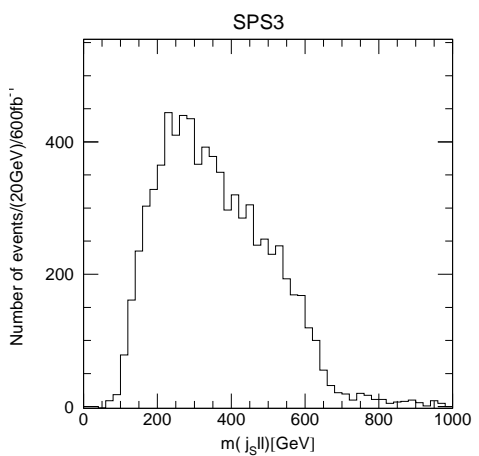

b)

FIG. 2: The $m\left(j_{S} l l\right)$ distributions for a) SPS1a and b) SPS3.
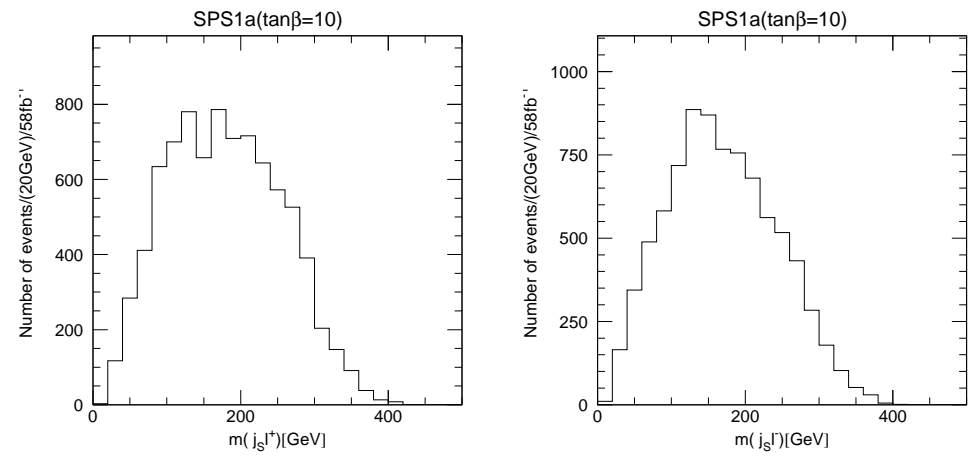

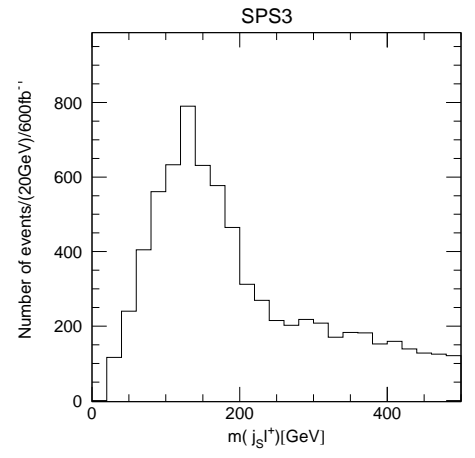

a)

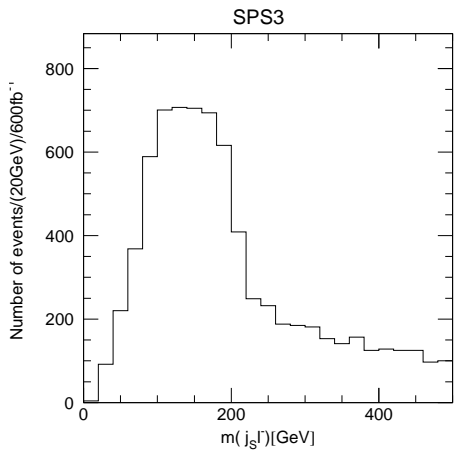

b)

FIG. 3: a) $m\left(j_{S} l^{+}\right)$and b) $m\left(j_{S} l^{-}\right)$distributions for SPS1a (the upper plots) and SPS3 (the lower plots). 
for SPS3, therefore the lepton from $\tilde{\chi}_{2}^{0}$ decay is less energetic. For SPS1a (SPS3) the edge structure is clearly seen in $j_{S} l^{+}\left(j_{S} l^{-}\right)$distribution. This is because the dominant mode is $l_{R}\left(l_{L}\right)$ for SPS1a (SPS3), therefore $l^{+}\left(l^{-}\right)$tend to go to the opposite direction to $q$ in the rest frame of $\tilde{q}$, respectively.

Fig. 4 a) shows the difference of the number of events after the background subtractions $N\left(j l^{ \pm}\right)$as the function of $m\left(j l^{ \pm}\right)$. The deviation from the zero are clearly seen. We also show b) the reconstructed charge asymmetry $\left[N_{\mathrm{sig}}\left(j l^{+}\right)-N_{\mathrm{sig}}\left(j l^{-}\right)\right] /\left[N_{\mathrm{sig}}\left(j l^{+}\right)+N_{\mathrm{sig}}\left(j l^{-}\right)\right]$ as a function of $m(j l)$ and c) the calculated charge asymmetry for $m(q l)$. The distribution of the measured asymmetry is diluted due to the $\tilde{q}^{*}$ productions and the decay and there are also the contamination of the events where a wrong jet is selected as $j_{S}$. However b) and c) are qualitatively similar. The distribution shows positive asymmetry near $m(j l)$ endpoint for SPS1a, while it shows negative asymmetry for the smaller endpoint of $m(j l)$ distributions for SPS3.

For these charge asymmetry plots, we add the difference for $m\left(j_{S} l\right)$ and for $m\left(j_{L} l\right)$ distributions, therefore each event is used twice for our analysis. This is not a problem because events which contribute to the asymmetry in $m\left(j_{S} l\right)$ distribution and in $m\left(j_{L} l\right)$ distribution must be statistically independent. Note a $j_{S}$ is more likely to be a wrong jet when $m\left(j_{S} l l\right)$ is much smaller than the end point. A significant fraction of $j_{L}$ 's also originates from $\tilde{q}$ decay. This can be seen in the $m\left(j_{L} l l\right)$ distribution at SPS1a, which shows a bump structure $\sim m(q l l, \max )=425 \mathrm{GeV}$ as in Fig. 5. In this paper, we require $m(j l l)<450(700)$ and and require $m(l l)<80(70) \mathrm{GeV}$ for SPS1a(SPS3) except Fig. 1, Fig. 2, and Fig. 15.

The statistical error of the asymmetry must be estimated based on the number of the odd sign leptons $N_{\text {OS }}$ rather than the number of events after $e \mu$ event subtraction $N_{\text {sig }}$. We can check the statistical significance of the charge asymmetry by calculating $\Delta \chi^{2} /$ n.d.f. from a constant distribution as

$$
\Delta \chi^{2}=\min _{\text {for } c} \sum_{m(j l) \text { bins }} \frac{\left[N_{\mathrm{sig}}\left(j l^{+}\right)-N_{\mathrm{sig}}\left(j l^{-}\right)-c\right]^{2}}{N_{\mathrm{OS}}\left(j l^{+}\right)+N_{\mathrm{OS}}\left(j l^{-}\right)},
$$

which is listed in Table $\mathrm{V}$. 

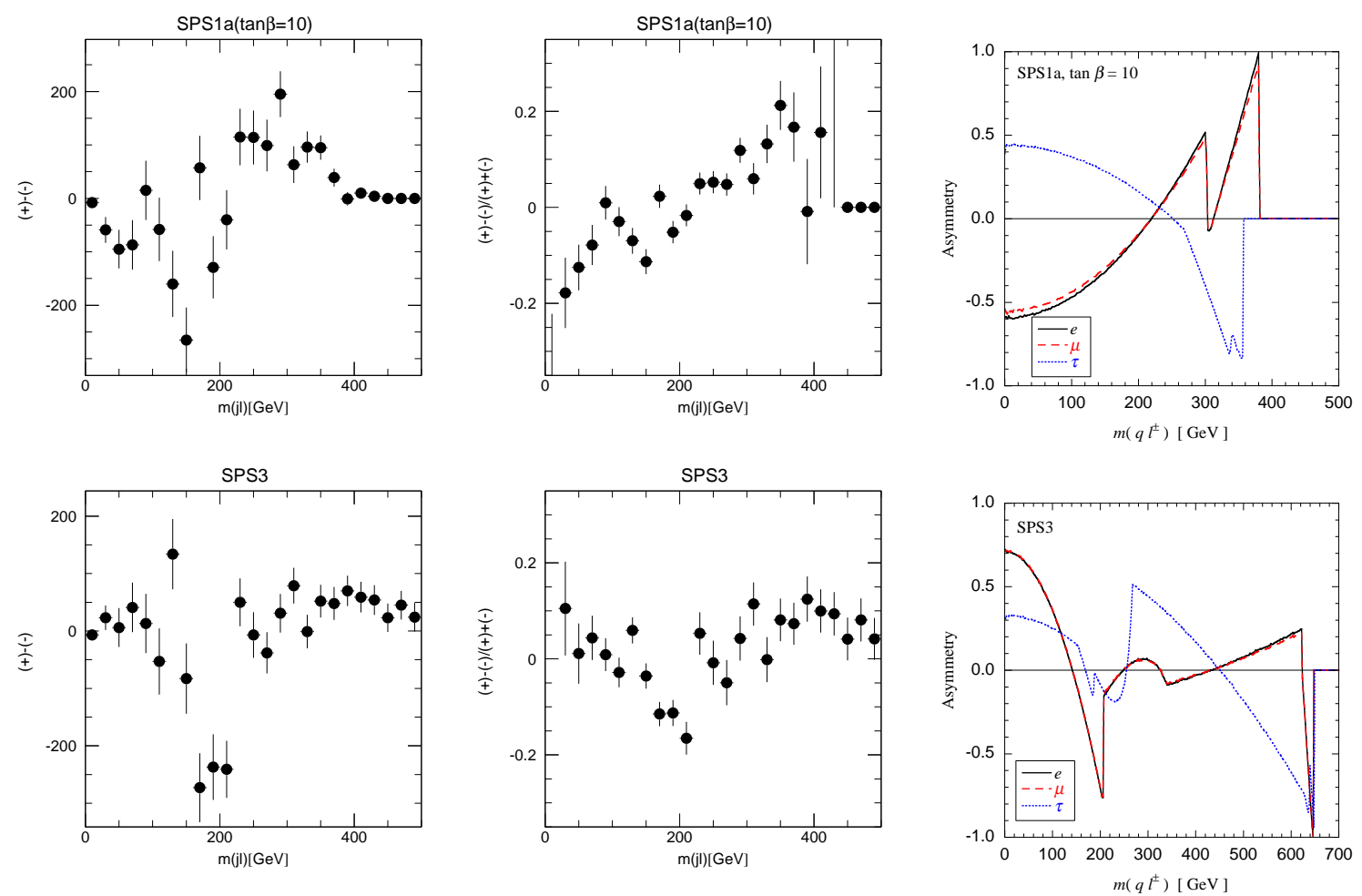

a)

b)

c)

FIG. 4: a) The difference between $m\left(j l^{+}\right)$and $m\left(j l^{-}\right)$distributions. b) The reconstructed asymmetry $\left[N_{\text {sig }}\left(j l^{+}\right)-N_{\text {sig }}\left(j l^{-}\right)\right] /\left[N_{\text {sig }}\left(j l^{+}\right)+N_{\text {sig }}\left(j l^{-}\right)\right]$. c) The calculated $\left[N\left(q l^{+}\right)-N\left(q l^{-}\right)\right] /\left[N\left(q l^{+}\right)+\right.$ $\left.N\left(q l^{-}\right)\right]$for $\tilde{q} \rightarrow q \tilde{\chi}_{2}^{0} \rightarrow q l^{\mp} \tilde{l}^{ \pm} \rightarrow q l^{\mp} l^{ \pm} \tilde{\chi}_{1}^{0}$ for $e^{+} e^{-}, \mu^{+} \mu^{-}$and $\tau^{+} \tau^{-}$. The differences for $j=j_{S}$ and $j=j_{L}$ are added. For $j=j_{L}$ we require $m\left(j_{L} l l\right)<m(q l l, \max )$.

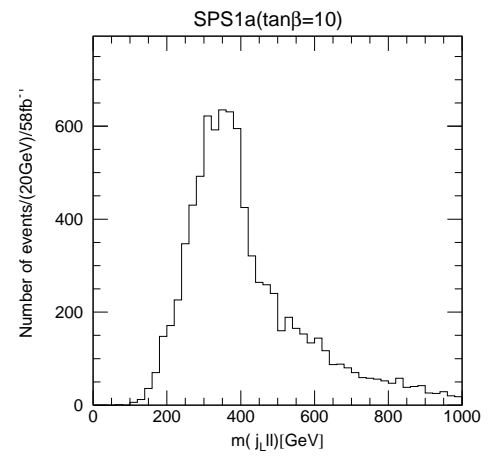

FIG. 5: The $m\left(j_{L} l l\right)$ distribution at SPS1a. 


\begin{tabular}{|c|c|c|c|c|c|c|c|}
\hline model points & $m_{\text {cut }}^{(\min )}$ & $m_{\text {cut }}^{(\max )}$ & $\begin{array}{c}N_{\mathrm{OS}}(j l \\
j_{S}\end{array}$ & $\begin{array}{c}N_{\mathrm{OS}}\left(j l^{-}\right) \\
j_{L}\end{array}$ & $\begin{array}{c}N_{\text {sig }}(j l \\
j_{S}\end{array}$ & $\begin{array}{c}N_{\text {sig }}\left(j l^{-}\right) \\
j_{L}\end{array}$ & $\begin{array}{c}S \\
\left(\Delta \chi^{2} / \text { n.d.f. }\right)\end{array}$ \\
\hline $\begin{array}{l}\operatorname{SPS} 1 \mathrm{a}(\tan \beta=10) \\
\left(e^{+} e^{-}+\mu^{+} \mu^{-}\right)\end{array}$ & 220 & 380 & 5426 & 7988 & 454 & 362 & $\begin{array}{c}7.37 \\
(84.9 / 15 \text { for } 60-380 \mathrm{GeV})\end{array}$ \\
\hline $\begin{array}{c}\text { SPS3 } \\
\left(e^{+} e^{-}+\mu^{+} \mu^{-}\right)\end{array}$ & 140 & 220 & 7691 & 8139 & -439 & -394 & $\begin{array}{c}6.43 \\
(51.9 / 7 \text { for } 60-220 \mathrm{GeV})\end{array}$ \\
\hline $\begin{array}{c}\operatorname{SPS} 1 \mathrm{a}(\tan \beta=20) \\
\left(e^{+} e^{-}\right)\end{array}$ & 220 & 380 & 3389 & 4574 & 174 & 99 & $\begin{array}{c}3.3 \\
(24.6 / 15 \text { for } 60-380 \mathrm{GeV})\end{array}$ \\
\hline
\end{tabular}

TABLE V: The statistical significance $S$ and $\Delta \chi^{2} /$ n.d.f. are estimated using the MC sample for SPS1a $(\tan \beta=10,20)$ and SPS3. For SPS1a $(\tan \beta=10)$ and SPS3, $3 \times 10^{6}$ events are used, while $15 \times 10^{6}$ events are used for $\operatorname{SPS} 1 \mathrm{a}(\tan \beta=20) \cdot N_{\mathrm{OS}}\left(j l^{ \pm}\right)$is the number of events with odd sign leptons with $m_{\text {cut }}^{(\min )}<m\left(j l^{ \pm}\right)<m_{\text {cut }}^{(\max )}$, while $N_{\text {sig }}\left(j l^{ \pm}\right)$are the number of events after $e \mu$ subtractions.

We define the statistical significance of the the charge asymmetry $S$ as

$$
\begin{aligned}
S^{2}= & \left.\frac{\left[N_{\mathrm{sig}}\left(j_{S} l^{+}\right)-N_{\mathrm{sig}}\left(j_{S} l^{-}\right)\right]^{2}}{N_{\mathrm{OS}}\left(j_{S} l^{+}\right)+N_{\mathrm{OS}}\left(j_{S} l^{-}\right)}\right|_{m_{\mathrm{cut}}^{\min }<m\left(j_{S} l\right)<m_{\mathrm{cut}}^{\max }} \\
& +\left.\frac{\left[N_{\mathrm{sig}}\left(j_{L} l^{+}\right)-N_{\mathrm{sig}}\left(j_{L} l^{-}\right)\right]^{2}}{N_{\mathrm{OS}}\left(j_{L} l^{+}\right)+N_{\mathrm{OS}}\left(j_{L} l^{-}\right)}\right|_{m_{\mathrm{cut}}^{\min }<m\left(j_{L} l\right)<m_{\mathrm{cut}}^{\max }} .
\end{aligned}
$$

where one of $m_{\text {cut }}$ is taken to the value of $m(j l)$ where the charge asymmetry changes its sign. We do not take into account the event outside the region $m_{\text {cut }}^{\min }<m(j l)<m_{\text {cut }}^{\max }$, where the asymmetry is opposite. If we add the contribution from both positive and negative asymmetry bins, we might overestimate the statistical significance because the $j l^{+}$and $j l^{-}$ distributions are of the same $j l l$ sample.

We list the statistical significance $S$ for SPS1a $(\tan \beta=10)$ and SPS3 for the sum of ee and $\mu \mu$ events in Table $\nabla$. The significance is based on the number of events for $\int \mathcal{L} d t \sim 60 \mathrm{fb}^{-1}$ for SPS1a $(\tan \beta=10)$ while it corresponds to $600 \mathrm{fb}^{-1}$ for SPS3. For $300 \mathrm{fb}^{-1}$ the left hand nature of slepton at SPS3 may still be seen with $S>3$. 


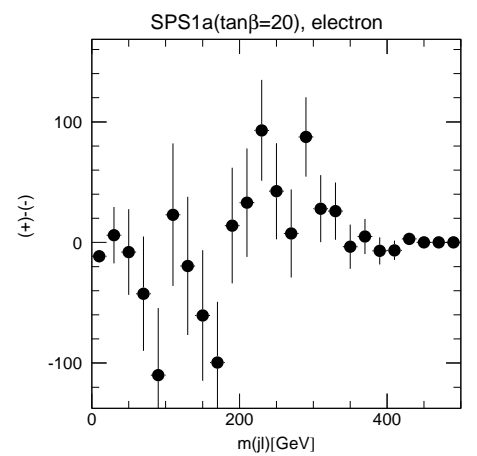

a)

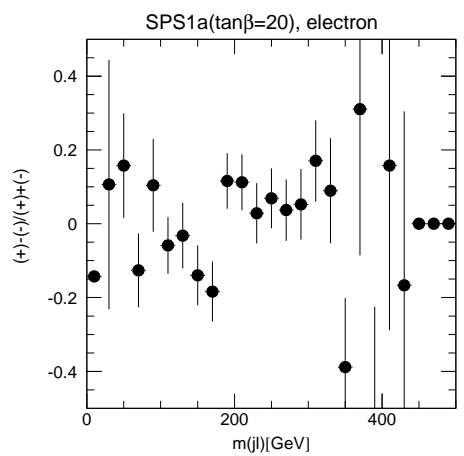

b)

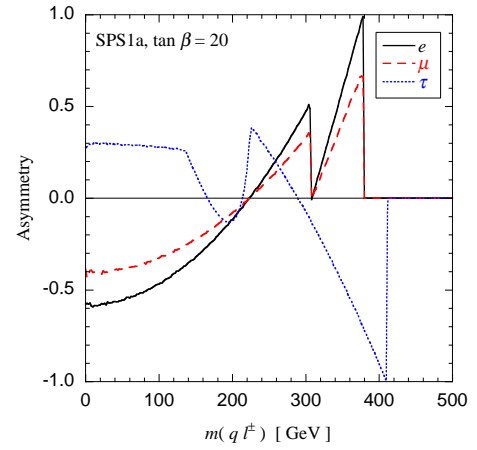

c)

FIG. 6: a) The reconstructed $N_{\mathrm{sig}}\left(j l^{+}\right)-N_{\mathrm{sig}}\left(j l^{-}\right)$as the function of $m(j l)$, and b) the charge asymmetry for $\int d t \mathcal{L}=300 \mathrm{fb}^{-1}$ at $\operatorname{SPS} 1 \mathrm{a}(\tan \beta=20)$. c) The charge asymmetry in the $m(q l)$ distribution at $\operatorname{SPS} 1 \mathrm{a}(\tan \beta=20)$.

\section{B. The non-universal effects}

In this subsection, we investigate the statistical significance of the non-universal effect between $e$ and $\mu$ for large $\tan \beta$. The deviation may be detectable with enough statistics because the systematics associated with the uncertainty of $\tilde{q}$ production and decay distributions are expected to cancel when one takes the $\mu / e$ ratio of the distributions. On the other side, the measurement will be extremely sensitive to systematic uncertainties on the relative efficiency and acceptance for electrons and muons.

The region of the parameters where we can expect large deviation is rather limited if we assume universal mass spectrum. This is because $\tilde{\tau}_{1}$ is lighter than the other sleptons and it is more $L-R$ mixed. $\tilde{\chi}_{2}^{0}$ decays into $\tilde{\tau}_{1}$ dominantly for large $\tan \beta$, and $\operatorname{Br}\left(\tilde{\chi}_{2}^{0} \rightarrow l \tilde{l}_{R}\right)$ $(l=e, \mu)$ is suppressed. As an example, $\operatorname{Br}\left(\tilde{\chi}_{2}^{0} \rightarrow \tilde{e} e\right)$, the number of accepted signal events and the total opposite sign two lepton events including backgrounds are listed for SPS1a $(\tan \beta=10,20)$ in Table VI for $60 \mathrm{fb}^{-1}$. Number of signal for $\tan \beta=20$ is reduced by a factor of 5 , while the number of backgrounds only reduced by a factor of 2 . The backgrounds dominantly come from $\tau$ leptons from Table VI, we can see that the statistical error of $e^{+} e^{-}$ratio $\Delta N(\operatorname{sig}) / N(\operatorname{sig}) \sim \sqrt{N_{\mathrm{OS}}} / N(\operatorname{sig}) \sim 0.02$ for $300 \mathrm{fb}^{-1}$. On the other hand, the statistical significance of the charge asymmetry is 3.3 at $\operatorname{SPS} 1 \mathrm{a}(\tan \beta=20)$. See Table $\mathrm{V}$ 


\begin{tabular}{|c||c|c|c|c|}
\hline point & $\operatorname{Br}\left(\tilde{\chi}_{2}^{0} \rightarrow e \tilde{e}\right)$ & $N_{\text {sig }}(e$ and $\mu)$ & $N_{\mathrm{OS}}$ & $\left(\frac{\operatorname{Br}(\mu)}{\operatorname{Br}(e)}-1\right)\left(\frac{\Delta N(\operatorname{sig})}{N(\mathrm{sig})}\right)^{-1}$ \\
\hline $\tan \beta=10$ & $6.3 \%$ & $1.39 \times 10^{4}$ & $2.68 \times 10^{4}$ & 5.4 \\
\hline $\tan \beta=20$ & $1.2 \%$ & $0.28 \times 10^{4}$ & $1.02 \times 10^{4}$ & 8.5 \\
\hline
\end{tabular}

TABLE VI: The $\operatorname{Br}\left(\tilde{\chi}_{2}^{0} \rightarrow e \tilde{e}\right)$ and accepted number of events for SPS1a $\tan \beta=10,20 . N_{\text {sig }}$ is the number of $l^{+} l^{-}$events after $e^{ \pm} \mu^{\mp}$ subtraction, while $N_{\mathrm{OS}}$ is the total odd sign two lepton events. The expected deviation of $\operatorname{Br}\left(\tilde{\chi}_{2}^{0} \rightarrow \tilde{\mu} \mu\right)$ from that for $\tilde{e} e$ is compared with the statistical error of the number of the $e^{+} e^{-}$events for $\int d t \mathcal{L}=300 \mathrm{fb}^{-1}$.

When $\tilde{\mu}$ left-right mixing is taken into account, $\operatorname{Br}(\mu) / \operatorname{Br}(e)=1.17$ and $A(\mu) / A(e)=$ 0.70 for $\tan \beta=20$ (Table [II). The statistical significance of the deviation of the branching ratio $[\operatorname{Br}(\mu) / \operatorname{Br}(e)-1] /[\Delta N(\operatorname{sig}) / N(\operatorname{sig})]$ is around 8.5 for $300 \mathrm{fb}^{-1}$. The significance of the deviation of charge asymmetry $S \times|(A(\mu)-A(e)) / A(e)|$ is only around 1 for $300 \mathrm{fb}^{-1}$.

The statistical significance at the LHC may be compared with that at the future linear collider (LC) at $\sqrt{s}=500 \mathrm{GeV}$, where $\tilde{\chi}_{2}^{0} \tilde{\chi}_{2}^{0}$ cross sections is $\sim 200 \mathrm{fb}^{-1}$ for a left handed beam [9]. There are $400 \tilde{\chi}_{2}^{0} \tilde{\chi}_{2}^{0}$ events followed by $\tilde{\chi}_{2}^{0} \rightarrow e \tilde{e}$ for $100 \mathrm{fb}^{-1}$ at SPS1a $(\tan \beta=20)$. Assuming no background, the deviation of branching ratios around $5 \%$ may be detectable.

Finally we discuss the decay distribution of $\tilde{\chi}_{2}^{0} \rightarrow \tau \tilde{\tau} \rightarrow \tau \tau \tilde{\chi}_{1}^{0}$. Ideally the signal distribution would show charge asymmetry near the end point as can be seen in Fig. 4 c). However, as a $\tau$ lepton decays into a meson and $\nu_{\tau}$ or $l \nu_{l} \nu_{\tau}$, one cannot measure the $\tau$ lepton momentum directly. When $\tau$ decays into leptons, the arising charged lepton is soft, and they typically have less than $1 / 3$ of the $\tau$ lepton energy. Such large smearing of the energy is not suitable for the charge asymmetry study. On the other hand, $\tau$ decay into a meson is a two body decay, and the meson might be detected as a low multiplicity isolated high $p_{T}$ jet. A QCD jet might also be mis-identified as a $\tau$ jet, and the probability is the function of $\tau$ tagging efficiency $\epsilon_{\tau}$ and $\tau$ jet $p_{T}$. QCD events become large background, however they can be removed by subtracting the sum of the distribution of $m\left(j_{\tau^{-}} j_{\tau^{-}}\right)$and $m\left(j_{\tau^{+}} j_{\tau^{+}}\right)$from that of $m\left(j_{\tau^{+}} j_{\tau^{-}}\right)$.

The identification of the hadronic $\tau$ decays has a strong dependence on the details of the detector performance. In the detector simulator ATLFAST, the detection efficiency of the $\tau$ jet and the rate of fake $\tau$ jet is estimated by using parameterizations of the full simulation data, which are described in Ref. 10]. However, the misidentification probability of the 
charge of the $\tau$ jet and the charge distribution of fake $\tau$ jets have not been fully accounted for in the parameterized simulation. We therefore do not study the charge asymmetry in $\tau$ jets in this paper.

\section{DISCUSSION}

In this paper, we study if SUSY study at the LHC can provide an information on the chiral nature of the sfermions. We especially study the charge asymmetry of $m\left(j l^{+}\right)$and $m\left(j l^{-}\right)$distribution which comes from the the decay cascade $\tilde{q} \rightarrow \tilde{\chi}_{2}^{0} \rightarrow \tilde{l} \rightarrow \tilde{\chi}_{1}^{0}$, where $\tilde{l}$ can be either $\tilde{l}_{R}$ or $\tilde{l}_{L}$.

When $\tilde{\chi}_{2}^{0}$ is wino like, the asymmetry $N\left(j l^{+}\right)-N\left(j l^{-}\right)$is negative near the $m(j l)$ end

point for pure $\tilde{l}_{L}$, while it is positive for pure $\tilde{l}_{R}$. We find that the charge asymmetry is significant for two representative mSUGRA points, SPS1a and SPS3, where slepton masses are lighter than $m_{\tilde{\chi}_{2}^{0}}$.

For $\tilde{\chi}_{2}^{0} \rightarrow \mu \tilde{\mu}_{1}$, the branching ratio and $j l$ distributions may depend on the relation among the left and right couplings of $\tilde{\chi}_{2}^{0} \tilde{\mu} \mu$ interactions. The branching ratio and charge asymmetry are proportional to $\left|L_{21}^{\mu}\right|^{2}+\left|R_{21}^{\mu}\right|^{2}$ and $\left|L_{21}^{\mu}\right|^{2}-\left|R_{21}^{\mu}\right|^{2}$, respectively. When $\tilde{\chi}_{2}^{0}$ is wino like, $L_{21}^{\mu}$ is proportional to small left-right mixing of $\tilde{\mu}$, and $\theta_{\mu}$, while $R_{21}^{\mu}$ is proportional to the small bino component $\left(U_{N}\right)_{21}$ of $\tilde{\chi}_{2}^{0}$. For large $\tan \beta, \cos \theta_{\mu}$ becomes comparable to $\left(U_{N}\right)_{21}$ in mSUGRA points, leading to the non-universalities in $\Gamma(l)$ and $A(l)$. We have shown that the LHC can detect $e-\mu$ non-universality at SPS1a(tan $\beta=10-20)$ for $\int d t \mathcal{L}=300 \mathrm{fb}^{-1}$ if detection efficiencies of $e$ and $\mu$ are understood at the LHC.

The discovery of supersymmetry and determination of the low energy Lagrangian are the goal of SUSY study at future colliders. So far, many physics studies have focused on the determination of SUSY parameters at benchmark points. On the other hand, the charge asymmetry in the $j l$ distribution provides an information on the chiral structure of the MSSM, which is constrained by nature of supersymmetry. Especially $\tilde{\mu}$ and $\tilde{e}$ nonuniversality is a direct evidence of $\tilde{\mu}_{L}-\tilde{\mu}_{R}$ mixing due to the $F$ term.

\section{Acknowledgments}

We thank the ATLAS collaboration members for useful discussions. We have made use 
of the physics analysis framework and tools which are the result of collaboration-wide efforts. We especially thank Dr. Polesello, Dr. Barr and Dr. Tovey for careful reading of the manuscript. This work is supported in part by the Grant-in-Aid for Science Research, Ministry of Education, Culture, Sports, Science and Technology (MEXT) of Japan (No. 11207101 and No. 15340076 for K. K. and No. 14540260 and No. 14046210 for M. M. N.). The works of T. G. and M. M. N. are supported in part by the Grant-in-Aid for the 21st Century COE "Center for Diversity and Universality in Physics" form the MEXT of Japan.

[1] I. Hinchliffe, F. E. Paige, M. D. Shapiro, J. Soderqvist and W. Yao, Phys. Rev. D 55, 5520 (1997) arXiv:hep-ph/9610544; S. Abdullin et al. [CMS Collaboration], J. Phys. G 28, 469 (2002) arXiv:hep-ph/9806366; ATLAS Collaboration, CERN-LHCC-99-14/15; H. Bachacou, I. Hinchliffe and F. E. Paige, Phys. Rev. D 62, 015009 (2000) arXiv:hep-ph/9907518; I. Hinchliffe and F. E. Paige, Phys. Rev. D 61, 095011 (2000) arXiv:hep-ph/9907519; B. C. Allanach, C. G. Lester, M. A. Parker and B. R. Webber, JHEP 0009, 004 (2000) arXiv:hep-ph/0007009.

[2] P. Richardson, JHEP 0111, 029 (2001) arXiv:hep-ph/0110108.

[3] A. J. Barr, arXiv:hep-ph/0405052.

[4] B. C. Allanach et al., in Proc. of the APS/DPF/DPB Summer Study on the Future of Particle Physics (Snowmass 2001) ed. N. Graf, Eur. Phys. J. C 25, 113 (2002) [eConf C010630, P125 (2001)] arXiv:hep-ph/0202233.

[5] M. Battaglia et al., Eur. Phys. J. C 22, 535 (2001) arXiv:hep-ph/0106204.

[6] H. Baer, F. E. Paige, S. D. Protopopescu and X. Tata, arXiv:hep-ph/0001086.

[7] G. Corcella et al., JHEP 0101, 010 (2001) arXiv:hep-ph/0011363.

[8] E. Richter-Was et al., ATLFAST2.21 ATLAS Internal Note PHYS-NO-079.

[9] K. Desch, J. Kalinowski, G. Moortgat-Pick, M. M. Nojiri and G. Polesello, JHEP 0402, 035 (2004) arXiv:hep-ph/0312069.

[10] I. Hinchliffe and F. E. Paige, Phys. Rev. D 63, 115006 (2001) arXiv:hep-ph/0010086.

[11] B. K. Gjelsten, E. Lytken, D. J. Miller, P. Osland, G. Polesello, "A detailed analysis of the measurement of SUSY masses with the ATLAS detector at the LHC", ATL-PHYS-2004-007. 